Ciência e Natura, v. 37 Part 2 2015, p. 320-327

ISSN impressa: 0100-8307 ISSN on-line: 2179-460X ciênciaenatura

\title{
Solution of the space-fractional telegraph equations by using HAM
}

Mehdi Abedi-Varaki ${ }^{1 *}$ Shahram Rajabi ${ }^{1}$, Vahid Ghorbani ${ }^{1}$, Farzad Hosseinzadeh ${ }^{1}$

${ }^{1}$ Sama technical and vocational training college, Islamic Azad University, Bandar Abbas branch, Bandar Abbas, Iran

${ }^{*}$ Crospending Author E-mail: Mehdi.Abedi75@yahoo.com

\begin{abstract}
In this study by using the Homotopy Analysis Method (HAM) obtained approximate solutions for the space and time-fractional telegraph equations. In Caputo sense (Yildirim, 2010)these equations considered. Examples are solved and the obtained results show to be more accurate than Adomian Decomposition Method (ADM) and are more efficient and commodious.
\end{abstract}

Keywords: Adomian Decomposition Method, Homotopy Analysis Method, time-fractional ttelegraph equation. 


\section{Introduction}

$\mathrm{T}$ he Homotopy Analysis Method (HAM) was initially proposed by Liao in his PhD thesis (Liao, 1992). A systematic and clear exposition on HAM is given in (Liao, 2003). In recent years, this method has been successfully employed to solve many types of nonlinear, homogeneous or non homogeneous, equations and systems of equations as well as problems in science and engineering (Liao, 1997). HAM contains a certain auxiliary parameter $\hbar$ which provides us with a simple way to adjust and control the convergence zone as well as the convergence rate of the series solution. Moreover, by means of the so-called $\hbar$ curve, it is easy to determine the valid ranges of $\hbar$ to obtain a convergent series solution. Thus, through HAM, an explicit analytic solution of nonlinear problems is possible. Over the last decades, fractional calculus has found diverse applications in various scientific and technological fields (Hilfer, 1999; Liao, 1995; Mainardi, 1997; Oldham, 1974; Podlubny, 1999). Fractional diffusion equations arise in continuous-time random walks modeling of anomalous diffusive and sub-diffusive systems, unification of diffusion and wave propagation phenomenon, and simplification of the results ( Agrawal, 2002). The nature of diffusion is characterized by temporal scaling of the meansquare displacement $\left\langle\mathrm{r}^{2}(\mathrm{t})\right\rangle \mathrm{t}^{\alpha}$. For standard diffusion, is equal to one, whereas in anomalous sub-diffusion $\alpha<1$ and in anomalous superdiffusion $\alpha>1$. Both of the anomalous diffusion processes have been unified in continuous time random walk models with spatial and temporal memories (Hilfer, 1999; Henry and Wearne, 2000; Kaya, 2000; Klafter et al. 1984). In this paper, we consider analytic and approximate solutions of the space-fractional telegraph equation of the form (Henry and Wearne, 2000):

$$
\frac{\partial^{\alpha} u}{\partial x^{\alpha}}=\frac{\partial^{2} u}{\partial x^{2}}+\frac{\partial u}{\partial t}+g(t), t \geq 0,0<\alpha \leq 2
$$

Subject to the initial and boundary conditions $\{u(x, 0)=s(x), 0<x<1\},\left\{u(0, \mathrm{t})=f_{1}(t), t \geq 0\right\},\left\{\frac{\partial u(0, t)}{\partial x}=f_{2}(t), t \geq 0\right\}(2)$

Where $\alpha$ is a parameter describing the order of the fractional space-derivative and $u(x, \mathrm{t})$ is assumed to be a causal function of space, i.e., vanishing for $x<0$. The fractional derivative is considered in Caputo sense (Podlubny, 1999). The general response expression contains a parameter describing the order of fractional

derivative that can be varied to obtain various responses. In the case of $\alpha=1$, fractional equation reduces to classical telegraph equation. We shall also examine the time-fractional telegraph equation, i.e.

$$
\frac{\partial^{2 \alpha} u}{\partial t^{2 \alpha}}+\lambda \frac{\partial^{\alpha} u}{\partial t^{\alpha}}=v \frac{\partial^{2} u}{\partial t^{2}},\{t>0,0<\alpha \leq 1\}
$$

Where $\lambda$ and $v$ are arbitrary constants and $u(x, \mathrm{t})$ is assumed to be a causal function of time vanishing for $t<0$. In the case of $\alpha=1$, timefractional equation reduces to classical telegraph equation.

\section{Basic definitions}

Definition 1. A real function $f(t), t>0$ is said to be space $C_{\alpha}, \alpha \in R$ if there exists a real number $p(>\alpha)$, such that $f(t)=t^{p} f_{1}(\mathrm{t})$ where $f_{1}(\mathrm{t}) \in[0, \infty)$. Clearly $C_{\alpha} \subseteq C_{\beta}$ if $\beta \leq \alpha$.

Definition 2. A function $f(t), t>0$ is said to be in the space $C_{\alpha}^{m}, m \in I N \cup 0$, if $f^{m} \in C_{\alpha}$.

Definition 3. The left sided Riemann-Liouville fractional integral of order $\mu \geq 0$, (Mainardi,1997; Luchko and Gorenflo, 1999; Moustafa, 2003) of a function $f \in C_{\alpha}, \alpha \geq-1$ is defined as

$$
\left\{\begin{array}{lr}
f(t) & \mu=0 \\
\frac{1}{\Gamma(\mu)} \int_{0}^{t} \frac{f(\tau)}{(t-\tau)^{(1-\mu)}} d(\tau) & \mu>0, t>0
\end{array}\right\}
$$

Definition 4. The (left sided) Caputo fractional derivative of $f, f \in C_{-1}^{m}, m \in I N \cup 0$, is defined as (Samko et al. 1993) 
$D_{*}^{\mu} f(t)=\frac{\partial^{\mu} f(t)}{\partial t^{\mu}}=\left\{\begin{array}{lr}\frac{\partial^{m} f(t)}{\partial t^{m}} & \mu=m \\ \frac{\partial^{m} f(t)}{\partial t^{m}} \mathrm{I}^{m-\mu} & m-1<\mu<m, m \in I N\end{array}\right\}$

Note that (Liao, 1992; Liao, 2003; Liao, 1997; Hilfer, 1999; Liao, 1995; Mainardi, 1997; Kaya,2000; Klafter et al. 1984; Luchko and Gorenflo, 1999; Moustafa, 2003)

$$
\begin{gathered}
I_{t}^{\mu} f(\chi, \mathrm{t})=\frac{1}{\Gamma(\mu)} \int_{0}^{t} \frac{f(\chi, \mathrm{s})}{(\mathrm{t}-\mathrm{s})^{(1-\mu)}} d s,\{\mu>0, \mathrm{t}>0\} \\
D_{*}^{\mu} f(t)=\mathrm{I}_{t}^{m-\mu} \frac{\partial^{m} f(\chi, t)}{\partial t^{m}} \quad\{m-1<\mu \leq m\} \\
I^{\mu} t^{\gamma}=\frac{\Gamma(\gamma+1)}{\Gamma(\gamma+\mu+1)} t^{\gamma+\mu},\{\mu>0, \gamma>-1, \mathrm{t}>0\} \\
\mathrm{I}^{\mu} t^{\gamma}=\frac{\Gamma(\gamma+1)}{\Gamma(\gamma+\mu+1)} t^{\gamma+\mu} \quad\{\mu>0, \gamma>-1, t>0\}
\end{gathered}
$$

Definition 5. The Mittag-Leffler function $E_{\alpha}(\mathrm{z})$ with $\alpha>0$ is defined by the following series representation, valid over the entire complex plane (Momani, 2005):

$$
E_{\alpha}(\mathrm{z})=\sum_{n=0}^{\infty} \frac{z^{n}}{\Gamma(\alpha n+1)}\{\alpha>0, \mathrm{z} \in \mathrm{C}\}
$$

\section{The homotopy analysis method}

In this study, we apply homotopy analysis method (Liao, 2005) in solving the stated problems. Consider fractional differential equations of form

$$
N(u(x, t))=0
$$

Subject to the following initial conditions:

$$
u_{k}(x, t)=C_{k}, k=1,2, \ldots, n
$$

Where $N$ are nonlinear operators that model the system, $x$ and $\mathrm{t}$ denote the independent variables and $u(x, t)$ are unknown functions. By means of generalizing the traditional homotopy method, Liao (Agrawal, 2002) constructed the so called zero-order deformation equations

$$
(1-p)\left[L\left(\Phi(x, t ; p)-u_{0}(x, t)\right]=p \hbar H(x, t) N \Phi(x, t ; p)\right.
$$

Where $p \in[0,1]$ is the embedding parameter, $\hbar \neq 0$ is the non-zero auxiliary functions and $L=D_{t}^{\alpha}(n-1<\alpha \leq n)$ is an auxiliary linear operator with the following property $L(\Phi(x, \mathrm{t}))=0$ when $\Phi(x, t)=0$.

$u_{0}(\mathrm{r}, \mathrm{t})$ is the initial estimates, of $u(x, \mathrm{t})$, and $u(x, t ; p)$ is the unknown functions, it is important, that one has great freedom in choosing auxiliary variables in HAM. Obviously, for $p=0$ and $p=1$ the follows holds, repesctively,

$$
\Phi(x, t ; 0)=u_{0}(x, \mathrm{t}), \Phi(x, t ; 1)=u_{0}(x, \mathrm{t})
$$

Thus, as $p$ increases from 0 to 1 , the solution $\Phi(x, t ; p)$ varies from initial guess $u_{0}(x, t)$ to the Solution $u(x, \mathrm{t})$.Taylor series expansion of $\Phi(x, t ; p)$ with respect to $p$, yields

$\Phi(x, t ; p)=u_{0}(x, t)+\sum_{m=1}^{\infty} u_{m}(x, t) p^{m}$

Where

$$
u_{m}(x, t)=\left.\left(\frac{1}{m !}\right) \frac{\partial^{m} \Phi(x, t ; p)}{\partial p^{m}}\right|_{p=0}
$$

If the auxiliary linear operator, the initial guess $u_{0}(x, t)$, the auxiliary parameter $\mathrm{I}^{\alpha}$, and the auxiliary functions are chosen properly, Eq.(12) converges for $p=1$, and we have

$$
u_{m}(x, t)=u_{0}(x, t)+\sum_{m=1}^{\infty} u_{m}(x, t)
$$

Define the vector

$$
u_{n}(x, t)=u_{0}(x, t), u_{1}(x, t), \ldots, u_{n}(x, t)
$$

Differentiating Eq. (11) $\mathrm{m}$ times with respect to the embedding parameter $p$, evaluating at $p=0$ and dividing by $\mathrm{m}$ !, yield the $m^{\text {th }}$ - order deformation equation

$$
\begin{aligned}
& L\left[u_{m}(x, t)-\chi_{m} u_{m-1}(x, t)\right]=\hbar H(x, t) R_{m}\left[u_{m-1}(x, t)\right] \\
& \mathrm{R}_{m}\left[u_{m-1}(x, t)\right]=\left.\left(\frac{1}{(m-1) !}\right) \frac{\partial^{m-1} N[\Phi(x, t ; p)]}{\partial p^{m-1}}\right|_{p=0}
\end{aligned}
$$

And

$$
\chi_{m}=\left\{\begin{array}{ll}
0 & m \leq 1 \\
1 & m>1
\end{array}\right\}
$$

Applying the Riemann-Liouville integral operator $\mathrm{I}^{\alpha}$ to both sides of Eq.(15), results the following

$$
u_{m}(x, t)=\chi_{m} u_{m-1}(x, t)-\chi_{m} \sum_{j=0}^{n-1} u_{m-1}^{(j)}\left(x, 0^{+}\right) \frac{t^{j}}{j !}+\hbar H(x, t) \mathrm{I}^{\alpha}\left[\mathrm{R}_{m}\left(u_{m-1}\right)\right.
$$

Suppose that using HAM, a series solution based on parameter $\hbar$ has been found; now, the question is, what values of $\hbar$ will ensure convergence for such series over a large enough zone with an acceptable rate of convergence. According to (Abbasbandy and Shirzadi, 2009) if one investigates the graph of solutions versus $\hbar$, for cases that a unique solution exists, acceptable values of $\hbar$ are those for which the series convergences to the same solution. Hence, there 
will be a flat line in such a graph signifying passable ranges of $\hbar$.

\section{4 space-fractional telegraph equations}

HAM is applied to systems modeled using the space and time-fractional telegraph equations.

Example 1. Consider the following system of space and time-fractional telegraph equations:

$$
\frac{\partial^{\alpha} u}{\partial x^{\alpha}}=\frac{\partial^{2} u}{\partial t^{2}}+\frac{\partial u}{\partial t}+u, t \geq 0,0<x \leq 2
$$

Subject to boundary conditions

$$
u(0, t)=e^{-t}, \frac{\partial u(0, t)}{\partial x}=e^{-t}, t \geq 0
$$

and initial condition

$$
u(x, 0)=1+x, 0<x<1
$$

Where $0<\alpha<2$ and for the special case when $\alpha=2$, this system represents a homogeneous

telegraph and has been solved successfully (Kaya, 2000). In applying the homotopy analysis method, based on initial conditions given in $\mathrm{Eq} \square(24)$, it is convenient to choose

$$
u_{0}(x, \mathrm{t})=e^{-t}(1+x)
$$

As an initial approximation of Eq.(23). The linear operator can be defined as

$$
L[\Phi(x, t ; p)]=\mathrm{D}_{* x}^{\alpha}[\Phi(x, t ; p)]
$$

With the property $L\left[c_{1}+c_{2} x\right]=0$, where $c_{i}=(i=1,2)$ are integral constants, and the nonlinear operator $N[\Phi(x, t ; p)]$ can be stated as

$$
\begin{aligned}
& N[\Phi(x, t ; p)]=\mathrm{D}_{* x}^{\alpha}[\Phi(x, t ; p)]-\frac{\partial^{2}}{\partial t^{2}}[\Phi(x, t ; p)] \\
& -\frac{\partial}{\partial t}[\Phi(x, t ; p)]-[\Phi(x, t ; p)]
\end{aligned}
$$

Using the above definitions, and assuming $H(x, t)=1$ the zero-order deformation equation can be constructed as

$$
(1-q) L\left[\Phi(x, t, p)-u_{0}(x, t)\right]=p \hbar N[(x, t ; p)]
$$

Obviously, for $p=0$ and $p=1$

$$
\Phi(x, t, 0)=u_{0}(x, t), \Phi(x, t, 1)=u(x, t)
$$

Respectively. The $m^{\text {th }}$-order deformation equations can then be written as

$$
L\left[u_{m}(x, t)-\chi u_{m-1}(x, t)\right]=\hbar R_{m}\left(u_{m-1}\right)
$$

Where

$$
R_{m}\left(u_{m-1}\right)=\mathrm{D}_{* x}^{\alpha} u_{m-1}-\frac{\partial^{2} u_{m-1}}{\partial t^{2}}-\frac{\partial u_{m-1}}{\partial t}-u_{m-1}
$$

Solution of the $m^{\text {th }}$-order deformation $\operatorname{Eq}(31)$ is,

$$
\begin{aligned}
& u_{m}(x, t)=\left(\chi_{m}+\hbar\right)\left[u_{m-1}(x, t)\right]-\left(\chi_{m}+\hbar\right) u_{m-1}(0, \mathrm{t}) \\
& -\left(\chi_{m}+\hbar\right)\left[\frac{\partial u_{m-1}(0, t)}{\partial x}\right]-\hbar I^{\alpha}\left[\frac{\partial^{2} u_{m-1}}{\partial t^{2}}+\frac{\partial u_{m-1}}{\partial t}+u_{m-1}\right]
\end{aligned}
$$

Since

$$
u(r, \mathrm{t})=u_{0}(x, t)+\sum_{m=1}^{\infty} u(r, t)
$$

And

$$
u_{0}(x, t)=\mathrm{e}^{-t}(1+x)
$$

Then,

$$
u_{1}(x, t)=-\hbar e^{t}\left[\frac{x^{\alpha}}{\Gamma(\alpha+1)}+\frac{x^{\alpha+1}}{\Gamma(\alpha+2)}\right]
$$

From (33) and (36)

$$
u_{m-1}^{(j)}(0, t)=0,\{j=0,1\}
$$

Thus

$$
\begin{aligned}
& u_{2}(x, t)=(1+\hbar) u_{1}(x, t)-\hbar e^{-t} u_{1}(x, t) \\
& -\hbar e^{-t}\left[\frac{x^{2 \alpha}}{\Gamma(2 \alpha+1)}+\frac{x^{2 \alpha+1}}{\Gamma(2 \alpha+2)}\right] \\
& u_{2}(x, t)=(1+\hbar) u_{2}(x, t)-\hbar e^{-t} u_{1}(x, t) \\
& -\hbar e^{-t}\left[\frac{x^{3 \alpha}}{\Gamma(3 \alpha+1)}+\frac{x^{3 \alpha+1}}{\Gamma(3 \alpha+2)}\right]
\end{aligned}
$$

Total solution can be written as:

$$
\begin{aligned}
& u_{2}(x, t)=(1+\hbar) u_{1}(x, t)-\hbar\left[(2+t) \frac{x^{2 \alpha}}{\Gamma(2 \alpha+1)}\right. \\
& \left.-(1+t) \frac{x^{3 \alpha}}{\Gamma(3 \alpha+1)}-\frac{2 x^{3 \alpha+2}}{\Gamma(3 \alpha+3)}\right] \\
& -\hbar(1+\hbar) e^{-t}\left[\frac{x^{\alpha}}{\Gamma(\alpha+1)}+\frac{x^{\alpha+1}}{\Gamma(\alpha+2)}\right. \\
& \left.+\frac{x^{2 \alpha}}{\Gamma(2 \alpha+1)}+\frac{x^{2 \alpha+1}}{\Gamma(2 \alpha+2)}+\ldots\right]
\end{aligned}
$$

And in the special case of $\hbar=1$, it yields

$$
\begin{aligned}
& u(x, t)=e^{-t}\left[1+x+\frac{x^{\alpha}}{\Gamma(\alpha+1)}+\frac{x^{\alpha+1}}{\Gamma(\alpha+2)}\right. \\
& \left.+\frac{x^{2 \alpha}}{\Gamma(2 \alpha+1)}+\frac{x^{2 \alpha+1}}{\Gamma(2 \alpha+2)}+\ldots\right]
\end{aligned}
$$

If $\alpha=2$, the solution given in (Kaya, 2000) is reproduced, i.e.

$$
u(x, t)=e^{-t}\left[1+x+\frac{x^{2}}{2 !}+\frac{x^{3}}{3 !}+\frac{x^{4}}{4 !}+\ldots\right]
$$


This solution is equivalent to the exact solution in closed from

$$
(x, t)=e^{(x-t)}
$$

Example 2 . Consider the following no homogeneous space-fractional telegraph equation:

$$
\begin{aligned}
& \frac{\partial^{\alpha} u}{\partial x^{\alpha}}=\frac{\partial^{2} u}{\partial t^{2}}+\frac{\partial u}{\partial t} \\
& +u-x^{2}-t+1,\{t \geq 0,0<\alpha \leq 2\}
\end{aligned}
$$

Subject to boundary conditions:

$$
u(0, t)=t, \frac{\partial u(0, t)}{\partial x}=0, t \geq 0
$$

And initial condition

$$
u(x, 0)=x^{2}, 0<x<1
$$

Where $0<\alpha<2$ and for the special case when $\alpha=2$, this system represents a homogeneous telegraph and is solved in (Kaya, 2000) as an application of homotopy analysis method. In view of the initial condition given by Eq.(24), it is convenient to define

$$
u_{0}(x, t)=t+I^{\alpha}\left[-x^{2}-t+1\right]
$$

As an initial approximation to Eq.(23). Let the linear operator

$$
L[\Phi(x, t ; p)]=\lambda \mathrm{D}_{* t}^{\alpha}[\Phi(x, t ; p)]
$$

With the property $L\left[c_{1}+c_{2} x\right]=0$, wherec $_{i}(i=1,2)$ are integral constants. The nonlinear operator can be written as

$$
\begin{aligned}
& N[\Phi(x, t ; p)]=\mathrm{D}_{* x}^{\alpha}[\Phi(x, t ; p)]-\frac{\partial^{2}}{\partial t^{2}}[\Phi(x, t ; p)] \\
& -\frac{\partial}{\partial t}[\Phi(x, t ; p)]-[\Phi(x, t ; p)]
\end{aligned}
$$

Using the above definition, along with the assumption that $H(x, t)=1$ the zero-order deformation equation can be constructed as

$$
\begin{aligned}
& (1-q) L[\Phi(x, t ; p) \\
& \left.-u_{0}(x, t)\right]=p \hbar N[\Phi(x, t ; p)]
\end{aligned}
$$

Obviously, for $\mathrm{p}=0$ and $\mathrm{p}=1$

$$
\Phi(x, t ; 0)=u_{0}(x, t), \Phi(x, t ; 1)=u(x, t)
$$

Respectively; thus, the $m^{\text {th }}$ - order deformation equations becomes

$$
L\left[u_{m}(x, t)-x_{m} u_{m-1}(x, t)\right]=\hbar R_{m}\left(u_{m-1}\right)
$$

Where

$$
R_{m}\left(u_{m-1}\right)=\mathrm{D}_{* x}^{\alpha} u_{m-1}-\frac{\partial^{2} u_{m-1}}{\partial t^{2}}-\frac{\partial u_{m-1}}{\partial t}-u_{m-1}
$$

Now the solution of this the $m^{\text {th }}-$ order deformation $\mathrm{Eq}(52)$,can be written as

$$
\begin{aligned}
& u(x, t)=t-\frac{2 x^{2 \alpha}}{\Gamma(\alpha+3)}+(1-t) \frac{x^{\alpha}}{\Gamma(\alpha+1)}+(1+t) \frac{x^{\alpha}}{\Gamma(\alpha+1)}- \\
& \left.t \frac{x^{2 \alpha}}{\Gamma(2 \alpha+1)}-\ldots\right]-\hbar(1+\hbar)\left[(1+t) \frac{x^{\alpha}}{\Gamma(\alpha+1)}-\right. \\
& \left.t \frac{x^{2 \alpha+2}}{\Gamma(2 \alpha+3)}+(2+t) \frac{x^{2 \alpha}}{\Gamma(2 \alpha+1)}-(1+t) \frac{x^{3 \alpha}}{\Gamma(3 \alpha+1)}+\ldots\right] \\
& -\left(\chi_{m}+\hbar\right)\left[\frac{\partial u_{m-1}(0, t)}{\partial x}\right]-\hbar I^{\alpha}\left[\left(1-\chi_{m}\right)\left(-x^{2}-t+1\right)\right]- \\
& \hbar I^{\alpha}\left[\frac{\partial^{2} u_{m-1}}{\partial t^{2}}+\frac{\partial u_{m-1}}{\partial t}+u_{m-1}\right]
\end{aligned}
$$

Finally

$$
u(x, t)=u_{0}(x, t)+\sum_{m=1}^{\infty} u_{m}(x, t)
$$

This yield

$$
\begin{aligned}
& u_{0}(x, t)=t+I^{\alpha}\left[\left(-x^{2}-t+1\right)\right]= \\
& t-\frac{2 x^{2+\alpha}}{\Gamma(\alpha+3)}+(1-t) \frac{x^{\alpha}}{\Gamma(\alpha+1)} \\
& u_{1}(x, t)=-\hbar I^{\alpha}\left[\frac{\partial^{2} u_{0}}{\partial t^{2}}+\frac{\partial u_{0}}{\partial t}+u_{0}\right]= \\
& \left.(1+t) \frac{x^{\alpha}}{\Gamma(\alpha+1)}-t \frac{x^{2 \alpha}}{\Gamma(2 \alpha+1)}-\frac{2 x^{2 \alpha+2}}{\Gamma(2 \alpha+3)}\right] \\
& u_{2}(x, t)=(1+\hbar) u_{1}(x, t)-\hbar\left[(2+t) \frac{x^{2 \alpha}}{\Gamma(2 \alpha+1)}-\right. \\
& \left.(1+t) \frac{x^{3 \alpha}}{\Gamma(3 \alpha+1)}-\frac{2 x^{3 \alpha+2}}{\Gamma(3 \alpha+3)}\right] \\
& u(x, t)=\hbar\left[\left(t-\frac{2 x^{2+\alpha}}{\Gamma(\alpha+3)}+(1-t) \frac{x^{\alpha}}{\Gamma(\alpha+1)}+\right.\right. \\
& \left.(1+t) \frac{x^{\alpha}}{\Gamma(\alpha+1)}-t \frac{x^{2 \alpha}}{\Gamma(2 \alpha+1)}-\ldots\right] \\
& -\hbar(1+\hbar)\left[(1+t) \frac{x^{\alpha}}{\Gamma(\alpha+1)}-\right. \\
& t \frac{2 x^{2 \alpha+2}}{\Gamma(2 \alpha+3)}+(2+t) \frac{x^{2 \alpha}}{\Gamma(2 \alpha+1)} \\
& \left.-(1+t) \frac{x^{3 \alpha}}{\Gamma(3 \alpha+1)}+\ldots\right]
\end{aligned}
$$

So in the special case $\hbar=1$, one obtains $u(x, t)$ :

$$
\begin{aligned}
& u(x, t)=t-\frac{2 x^{2 \alpha}}{\Gamma(\alpha+3)}+(1-t) \frac{x^{\alpha}}{\Gamma(\alpha+1)}+ \\
& (1+t) \frac{x^{\alpha}}{\Gamma(\alpha+1)}-t \frac{x^{2 \alpha}}{\Gamma(2 \alpha+1)}-\ldots
\end{aligned}
$$


It can be seen that the self-canceling noise terms appear between various components. Setting $\alpha=2$, and canceling the noise terms in (59) the exact solution for this special case is obtained, i.e.

$$
u(x, t)=x^{2}+t
$$

\section{Time-fractional telegraph equations}

Consider the time-fractional telegraph equation,

$\frac{\partial^{2 \alpha} u}{\partial t^{2 \alpha}}+\lambda \frac{\partial^{\alpha} u}{\partial t^{\alpha}}=v \frac{\partial^{2} u}{\partial t^{2}},\{t>0,0<\alpha \leq 1\}$

Subject to initial boundary and conditions $u(x, 0)=\mathrm{g}_{1}(x), u_{t}(x, t)=g_{2}(x), u(0, t)=s(t)(62)$

Let

$$
u_{0}(x, t)=\mathrm{g}_{1}(x)+\mathrm{g}_{2}(x) t
$$

As an initial approximation to solution of $\mathrm{Eq}(23)$. Defining the linear operator

$$
L[\Phi(x, t ; p)]=\mathrm{D}_{* x}^{2 \alpha}[\Phi(x, t ; p)]
$$

With

property $L\left[c_{1}+c_{2} x\right]=0$, where $c_{i}(i=1,2)$ are integral constants, and the nonlinear operator

$$
\begin{aligned}
& N[\Phi(x, t ; p)]=\mathrm{D}_{* t}^{2 \alpha}[\Phi(x, t ; p)]+ \\
& \lambda \mathrm{D}_{* t}^{\alpha}[\Phi(x, t ; p)]-v \frac{\partial^{2}}{\partial x^{2}}[\Phi(x, t ; p)]
\end{aligned}
$$

Along with the assumption that $H(x, t)=1$ the zero-order deformation equation is constructed as

$$
\begin{aligned}
& (1-q) L[\Phi(x, t ; p) \\
& \left.-u_{0}(x, t)\right]=p \hbar N[\Phi(x, t ; p)]
\end{aligned}
$$

Noting that for $p=0$ and $p=1$

$$
\Phi(x, t, 0)=u_{0}(x, t), \Phi(x, t, 1)=u(x, t)
$$

Respectively; thus, the $m^{\text {th }}$ - order deformation equations, becomes

$$
L\left[u_{m}(x, t)-x_{m} u_{m-1}(x, t)\right]=\hbar R_{m}\left(u_{m-1}\right)
$$

Where

$$
R_{m}\left(u_{m-1}\right)=D_{* t}^{2 \alpha}\left[u_{m-1}\right]+\lambda D_{* t}^{\alpha}\left[u_{m-1}\right]-v \frac{\partial^{2}}{\partial x^{2}}\left[u_{m-1}\right]
$$

Solution of the $m^{\text {th }}$ - order deformation, $\operatorname{Eq}(68)$, can be written as

$$
\begin{aligned}
& u_{m}(x, t)=\left(\chi_{m}+\hbar\right) \\
& {\left[u_{m-1}(x, t)\right]-\left(\chi_{m}+\hbar\right)\left[\sum_{j=0}^{1} u_{m-1}^{(j)}(x, 0) \frac{t^{j}}{j !}\right]} \\
& +\hbar I^{2 \alpha}\left[\lambda \mathrm{D}_{* t}^{\alpha}\left[u_{m-1}\right]-v \frac{\partial^{2}}{\partial x^{2}}\left(u_{m-1}\right)\right]
\end{aligned}
$$

Homotopy Analysis Method assumes a series solution for $u(x, t)$ of the form

$u(x, t)=u_{0}(x, t)+\sum_{m=1}^{\infty} u_{m}(x, t)$

From Eq.(70) $u_{i}(x, t)$ terms are obtained successively

$$
\begin{aligned}
& u_{0}(x, t)=g_{1}(x)+g_{2}(x) t \\
& u_{1}(x, t)=\hbar I^{2 \alpha}\left[\lambda D_{* t}^{\alpha}\left[u_{0}\right]-v \frac{\partial^{2}}{\partial x^{2}}\left[u_{0}\right]\right] \\
& =\hbar \lambda\left[g_{1} \frac{t^{\alpha}}{\Gamma(\alpha+1)}+g_{2} \frac{t^{\alpha+1}}{\Gamma(\alpha+2)}\right] \\
& -\hbar v\left[g_{1}^{(2)} \frac{t^{2} \alpha}{\Gamma(2 \alpha+1)}+g_{2}^{(2)} \frac{t^{2 \alpha+1}}{\Gamma(2 \alpha+2)}\right]
\end{aligned}
$$

$$
\begin{aligned}
& u_{2}(x, t)=(1+\hbar) \hbar \lambda\left[g_{1} \frac{t^{\alpha}}{\Gamma(\alpha+1)}+g_{2} \frac{t^{\alpha+1}}{\Gamma(\alpha+2)}\right] \\
& -\hbar\left[\left(\lambda^{2} g_{1}+(1+\hbar) v g_{1}^{(2)}\right)\right. \\
& -\hbar\left[\left(\lambda^{2} g_{2}+(1+\hbar) v g_{2}^{(2)} \frac{t^{2 \alpha+1}}{\Gamma(2 \alpha+2)}\right]\right. \\
& +2 \hbar v \lambda\left[g_{1}^{(2)} \frac{t^{3} \alpha}{\Gamma(3 \alpha+1)}+g_{2}^{(2)} \frac{t^{3 \alpha+1}}{\Gamma(3 \alpha+2)}\right] \\
& -\hbar v^{2}\left[g_{1}^{(4)} \frac{t^{4} \alpha}{\Gamma(4 \alpha+1)}+g_{2}^{(4)} \frac{t^{4 \alpha+1}}{\Gamma(4 \alpha+2)}\right]
\end{aligned}
$$

Finally

$$
\begin{aligned}
& u(x, t)=\mathrm{g}_{1}(x)+\operatorname{tg}_{2}(x)+(2+\hbar) \hbar \lambda\left[\mathrm{g}_{1} \frac{t^{\alpha}}{\Gamma(\alpha+1)}+g_{2} \frac{t^{\alpha+1}}{\Gamma(\alpha+2)}\right] \\
& -\hbar\left[\left(\lambda^{2} \mathrm{~g}_{1}+(2+\hbar) v \mathrm{~g}_{1}^{(2)} \frac{t^{2} \alpha}{\Gamma(2 \alpha+1)}\right)-\hbar\left[\left(\lambda^{2} \mathrm{~g}_{2}+(2+\hbar) v g_{2}^{(2)} \frac{t^{2 \alpha+1}}{\Gamma(2 \alpha+2)}\right]\right.\right. \\
& +2 \hbar v \lambda\left[g_{1}^{(2)} \frac{t^{3} \alpha}{\Gamma(3 \alpha+1)}+g_{2}^{(2)} \frac{t^{3 \alpha+1}}{\Gamma(3 \alpha+2)}\right] \\
& -\hbar v^{2}\left[\mathrm{~g}_{1}^{(4)} \frac{t^{4} \alpha}{\Gamma(4 \alpha+1)}+\mathrm{g}_{2}^{(4)} \frac{t^{4 \alpha+1}}{\Gamma(4 \alpha+2)}\right]+\ldots
\end{aligned}
$$

Setting $\alpha=1$ and $\hbar=1$, we obtain:

$$
\begin{aligned}
& u(x, t)=g_{1}+t g_{2}-\lambda\left[g_{1} \frac{t^{\alpha}}{\Gamma(\alpha+1)}+g_{2} \frac{t^{\alpha+1}}{\Gamma(\alpha+2)}\right] \\
& +v\left[g_{1}^{(2)} \frac{t^{2 \alpha}}{\Gamma(2 \alpha+1)}+\right. \\
& \left.2 g_{2}^{(2)} \frac{t^{2 \alpha+1}}{\Gamma(2 \alpha+2)}\right]-2 v \lambda\left[g_{1}^{(2)} \frac{t^{3 \alpha}}{\Gamma(3 \alpha+1)}+3 g_{2}^{(2)} \frac{t^{3 \alpha+1}}{\Gamma(3 \alpha+2)}\right] \\
& +v^{2}\left[g_{1}^{(4)} \frac{t^{4 \alpha}}{\Gamma(4 \alpha+1)}+g_{2}^{(4)} \frac{t^{4 \alpha+1}}{\Gamma(4 \alpha+2)}\right]+\ldots
\end{aligned}
$$


Fig. 1 shows that interval $[-3,2]$ is " passable region" ; i.e. the series will converge for all values of $\hbar$ over this interval.

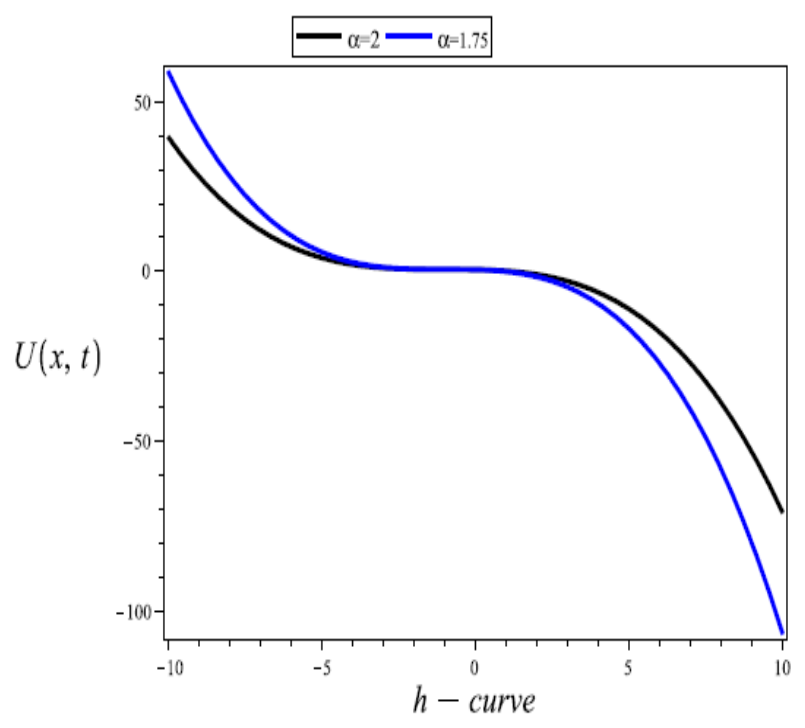

Figure1: $\hbar$ of $U(x, t)$ for $x=0.5, t=1$ $\alpha=2$ and $\alpha=1.75$

\section{Conclusions}

The HAM analysis method is a simple technique that offers two mechanisms, i.e. $\hbar$ and the auxiliary function $H(x, t)$, which allow the control of convergence rate within a specified convergence zone. This method yields the solution in a series form. An important property of this method is that it offers closed form solutions to nonlinear problems. The method is also suitable for handling differential systems with fractional derivatives. In this paper HAM was used to solve space-fractional telegraph equation. For value of $\alpha=2$, (see (Kaya, 2000; Momani, 2005)) exact solution was obtained. Homotopy Analysis Method is better than Adomian Decomposition Method in that it contains better approximations. (ADM) is a special case of (HAM) for which $H(x, t)=1$ and $\hbar=1$. Here for values of $0<\alpha \leq 2$ the auxiliary parameter $\hbar$ and auxiliary function $H(x, t)$ are selected appropriately in constructing the series solution. Examples are solved and the obtained results show to be more accurate than (ADM)(see ( Momani, 2005)).

\section{Acknowledgment}

The authors would like to thank Dr. Alireza Ahmadi from graduate university of advanced technology Kerman, Iran, for his helpful suggestion throughout this work.

\section{References}

Abbasbandy, S., Shirzadi, A. (2009). The Series Solution of Problem in the Calculus of Variations Via Homotopy Analysis Method. Z. Naturforsch, vol.64a, pp. 30-36.

Agrawal, Om P. (2002). Solution for a fractional diffusion-wave equation defined in a bounded domain. Nonlinear Dynamics, vol. 29, pp. 145-155.

Henry, B.I., Wearne, S.L. (2000). Fractional reaction-diffusion. Physica A, pp. 45-48.

Hilfer, R. (1999). Applications of fractional calculus in physics. Orlando: Academic Press.

Kaya, D. (2000). A new approach to the telegraph equation: An application of the decomposition method, Bull. Inst. Math., Acad. Sin, vol. 28, pp. 51-57.

Klafter,J., Blumen , A., Shlesinger, M.F. (1984). Fractal behavior in trapping and reaction: A random walk study, J. Stat. Phys, vol. 36, pp. 561-578.

Liao, SJ. (1992). The proposed homotopy analysis technique for the solution of nonlinear problems. PhD thesis, Shanghai Jiao Tong University.

Liao, S.J. (1995). An approximate solution technique which does not depend upon small parameters: a special example. Int J Nonlinear Mech, vol. 30, pp. 18-30.

Liao, S.J. (1997). Homotopy analysis method: a new analytical technique for nonlinear problems. Commun Nonlinear Sci Numer Simulat, vol. 2, pp. 95-100..

Liao, S.J. (2003). Beyond perturbation: introduction to the homotopy analysis method. Boca Raton:CRC Press, Chapman and Hall.

Liao, S.J. (2005), Int. J. Heat Mass Transfer, vol. 48, pp.25-29. 
Luchko Yu, Gorenflo R. (1997). An operational method for solving fractional differential equations with the Caputo derivatives. Acta. Math Vietnamica vol. 24, pp.20-33.

Mainardi, F. (1997). Fractional calculus: some basic problems in continuum and statistical mechanics. In: carpinteri A, Mainardi F, editors. Fractals and fractional calculus in continuum mechanics. New York: Springer-Verlag, pp. 291348.

Momani, S. (2005). Analytic and approximate solutions of the space and time-fractional telegraph equations, Applied Mathematics and Computation, vol.170, pp. 1126-1134

Moustafa, OL. (2003). On the Cauchy Problem for some fractional order partial differential equations. Chaos Soltions Fractals, vol. 18, pp. $35-40$.

Oldham, KB. (1974). Fractional differential calculus. New York: Academic Press.

Podlubny, I. (1999). Spanier J. The fractional equations. San Diego: Academic Press.

Samko, G., Kilbas, A.A., Marichev, OI. (1993). Fractional integrals and derivatives: theory and applications. Yverdon: Gordon and Breach.

Yildirim, A. (2010). Int. J. Comput. Mathematics, taylor \& francis, vol. 87, pp. 299-300. 